\title{
Online C-arm calibration using a marked guide wire for 3D reconstruction of pulmonary arteries
}

Étienne Vachon, Joaquim Miró, Luc Duong

Étienne Vachon, Joaquim Miró, Luc Duong, "Online C-arm calibration using a marked guide wire for 3D reconstruction of pulmonary arteries," Proc. SPIE 10135, Medical Imaging 2017: Image-Guided Procedures, Robotic Interventions, and Modeling, 101351V (3 March 2017); doi: $10.1117 / 12.2254175$ 


\title{
Online C-arm calibration using a marked guide wire for 3D reconstruction of pulmonary arteries
}

\author{
Étienne Vachon ${ }^{\mathrm{a}}$, Joaquim Mirób ${ }^{\mathrm{b}}$ and Luc Duong ${ }^{\mathrm{a}}$

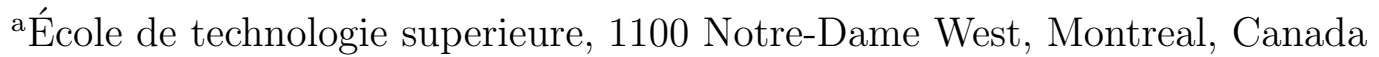 \\ ${ }^{\mathrm{b}}$ CHU Sainte-Justine, 3145 Côte-Sainte-Catherine, Montreal, Canada
}

\begin{abstract}
$3 \mathrm{D}$ reconstruction of vessels from 2D X-ray angiography is highly relevant to improve the visualization and the assessment of vascular structures such as pulmonary arteries by interventional cardiologists. However, to ensure a robust and accurate reconstruction, C-arm gantry parameters must be properly calibrated to provide clinically acceptable results. Calibration procedures often rely on calibration objects and complex protocol which is not adapted to an intervention context. In this study, a novel calibration algorithm for C-arm gantry is presented using the instrumentation such as catheters and guide wire. This ensures the availability of a minimum set of correspondences and implies minimal changes to the clinical workflow. The method was evaluated on simulated data and on retrospective patient datasets. Experimental results on simulated datasets demonstrate a calibration that allows a 3D reconstruction of the guide wire up to a geometric transformation. Experiments with patients datasets show a significant decrease of the retro projection error to $0.17 \mathrm{~mm} 2 \mathrm{D}$ RMS. Consequently, such procedure might contribute to identify any calibration drift during the intervention.
\end{abstract}

Keywords: X-ray - Fluoroscopy; X-ray imaging applications; Cardiac imaging and image analysis

\section{INTRODUCTION}

Congenital Heart Defects (CHD) are the most common type of birth defect and occur in 0.5 - 0.8 live births affecting 1.5 million children worldwide. Pulmonary stenosis is encountered in $10 \%$ of patients affected by another CHD. To diagnose and treat such diseases, percutaneous intervention is often preferred over open-heart surgery. A catheter is guided to the intervention area, where a contrast agent is injected, revealing the arteries in the X-ray images. This intervention is frequently conducted under biplane 2D X-ray guidance. This allows cardiologists to have a better visualization of arteries and vessels and to accurately evaluate relative 3D depths. Though, only experienced physicians are able to properly associate the information of the different views, but their analysis still involves a high intra- and inter-observer variability. ${ }^{1}$ An accurate $3 \mathrm{D}$ reconstruction could provide a repeatable and comprehensive visualization of the vessels geometry, ease measurements and permit simulations such as stent placement.

In this study, we aimed at proposing a simple workflow for improving C-arm calibration for pulmonary interventions, which is usually the first step of the reconstruction process. The importance of C-arm calibration has been widely documented for $3 \mathrm{D}$ reconstruction of vascular structures such as coronary arteries using $2 \mathrm{D}$ angiography; ${ }^{2-4}$ however, to our knowledge, no work has been done regarding the particularities of a reconstruction involving the major pulmonary arteries, which are significantly different in size and shape. Self-calibration methods, using the actual targeted anatomical structures were proposed for usage during the intervention. This is appealing for coronary arteries, since they display many identifiable bifurcations points for stereo correspondence, but for larger vessels, matching points can be challenging to identify properly. Also, a precisely manufactured calibration phantom as suggested by Rougee et al. ${ }^{5}$ and Kerrien et al. ${ }^{6}$ could yield more repeatable and robust results, however physicians considers that they would disturb the clinical protocol and interfere due to the occlusion it may produce over the targeted vascular structure. ${ }^{2}$

The proposed workflow uses a marked guide wire or catheter as a calibration object to identify correspondences across views. This is very appealing since marked guide wires are used routinely in the clinical workflow during treatment of pulmonary arteries intervention, are of known dimensions and are manufactured with accurate measurements. The embedded markers can ensure the availability of a minimum of correspondences between the 


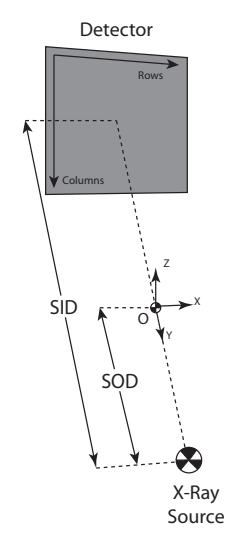

(a)

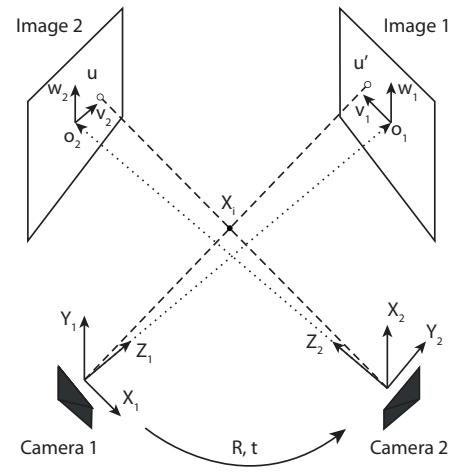

(b)

Figure 1: C-arm camera model : (a) relation between the X-ray source, the principal point and the detector, (b) geometric relationship between the Xray system

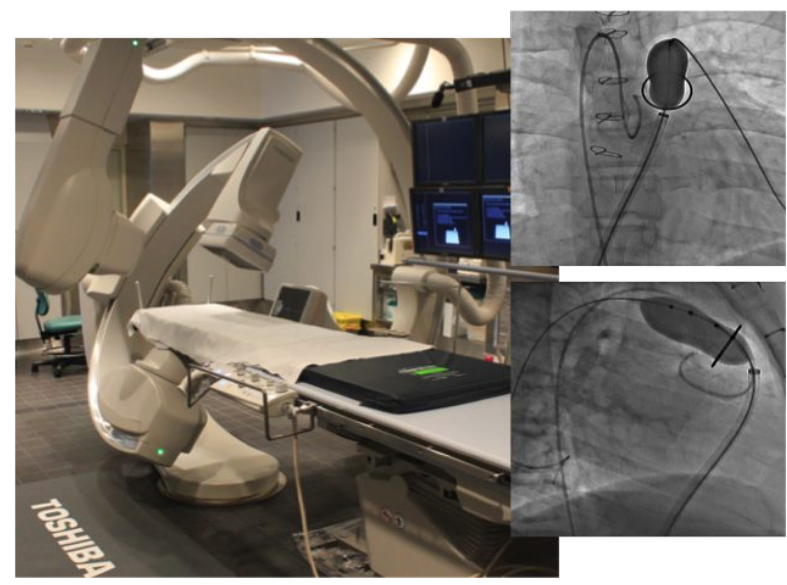

Figure 2: Actual biplane C-arm system: two radiographic sources are acquiring $\mathrm{X}$-ray images from two different angles for navigation guidance. Fluoroscopic sequences (in insert) depict the expansion of a balloon to treat a stenosis of a pulmonary artery.

projections and can also be used to infer others correspondences along the guide wire. This paper is organized as follow: first, an overview of the mathematical model of a biplane C-arm X-ray system is presented in Section 2.1. Second, Section 2.2 presents the stereo correspondence algorithm across biplane views. In section 2.3 we described an iterative calibration process. Finally, experimental results from simulated and retrospective patient datasets are presented in Section 3 and a conclusion and future works are presented in Section 4.

\section{METHODS}

\subsection{Mathematical model}

Generally, X-ray C-arm systems are modeled using the projective geometry of a pin-hole camera model, which is widely documented in the computer vision literature. ${ }^{3,7}$ In the X-ray model (1), the image is not inverted and the projection $\left(c_{x}, c_{y}\right)^{T}$ of the principal point $\mathbf{O}$ is not necessarily at the origin of the image $\left.\left(\left(c_{x}, c_{y}\right)\right)^{T}=(0,0)^{T}\right)$. Also, the pinhole model assumes that the coordinates of an image are Euclidean measures of the same horizontal and vertical scale, while in reality, the coordinates are in pixel whose shape is not necessarily square. The projection process is mathematically formulated through a combination of intrinsic and extrinsic parameters. The intrinsic parameters contained in the homogeneous matrix $K(1)$ encompass the focal length where $f_{x}=S I D / \alpha_{x}$ and $f_{y}=S I D / \alpha_{y}$, the skew $(s)$ accounting for the non-orthogonality of the axes, and the position of the principal point.

$$
K_{i}=\left[\begin{array}{cccc}
\frac{S I D_{i}}{\alpha_{x, i}} & s & c_{x, i} & 0 \\
0 & \frac{S I D_{i}}{\alpha_{y, i}} & c_{y, i} & 0 \\
0 & 0 & 1 & 0
\end{array}\right], i=1,2
$$

SID is the Source Image Distance, $\alpha_{x}$ and $\alpha_{y}$ are the Imager Pixel Spacing or the vertical and horizontal distance between two pixels. The extrinsic parameters define the position $t$ and orientation $R$ of a camera in relation to the world. Where $t$ is a $3 \times 1$ translation vector and $R$ a $3 \times 3$ rotation matrix. In a biplane system, it is convenient to define the world coordinates with reference to one of the cameras. Hence, the extrinsic parameters of the second camera directly define the relationship between the two views. Therefore, the extrinsic parameters 
of the first radiographic view are given by $[I \mid \mathbf{0}]$ where $I$ is a $3 \times 3$ identity matrix and $\mathbf{0}$ is the null vector and $[R \mid \mathbf{t}]$ represents the extrinsic parameters of the second view, with $R$ defined as

$$
R=R_{y}\left(-\theta_{2}\right) R_{x}\left(-\phi_{2}\right) R_{y}\left(\theta_{1}\right) R_{x}\left(\phi_{1}\right)
$$

where $R_{x}$ and $R_{y}$ respectively represent a rotation around the $x$ or $y$ axis in the Euclidean space. $\theta_{1}$ and $\theta_{2}$ are the Positioner Primary Angle defining a rotation in patient space in the Left Anterior Oblique or Right Anterior Oblique directions and $\phi_{1}$ and $\phi_{2}$ are rotations in the cranial or caudal direction. The translation vector $t$ is formulated as

$$
t=-R\left(\left[\begin{array}{lll}
0 & 0 & S O D_{1}
\end{array}\right]^{T}-R^{T}\left[\begin{array}{lll}
0 & 0 & S O D_{2}
\end{array}\right]^{T}\right) .
$$

where $S O D$ (Source Object Distance) is the distance between the X-ray source and the patient as estimated by the C-arm system. Then, the intrinsic and extrinsic parameters can be combined in order to form the projection matrices $P_{1}$ and $P_{2}$ which allow the projection of a space point $\mathbf{X}$ to the two views formulated as

$$
\mathbf{x}_{\mathbf{1}}=P_{1} \mathbf{X}=K_{1}[I \mid \mathbf{0}] \mathbf{X}, \mathbf{x}_{\mathbf{2}}=P_{2} \mathbf{X}=K_{2}[R \mid t] \mathbf{X}
$$

where $\mathbf{x}_{\mathbf{1}}$ and $\mathbf{x}_{\mathbf{2}}$ are the image coordinate of the projected $3 \mathrm{D}$ point.

\subsection{Shortest path matching}

$\mathrm{X}$-ray sequences were acquired during navigation for treatment of pulmonary stenosis using a Infinix-CFI BP CArm from Toshiba as illustrated in Fig. 2. Images were preprocessed to extract the guide wire. First, we applied a coherence enhancing diffusion filter to reduce the effect of radiographic noise. ${ }^{8}$ Then, a multiscale Hessian-based filter ${ }^{9}$ was applied to outline the shape of the instrument followed by a connected component analysis which was used to retain the largest connected component. The shape of the guide wire was finally retrieved using the guide wire centerline computed from flux-ordered homotopic thinning. ${ }^{10}$ If necessary, segmentation errors were corrected manually to obtain a perfect segmentation of the guide wire. Marked beads were manually identified on the images as known references. Stereo correspondence between views was established using a mapping between the centerlines of the guide wire from both views. Since this mapping is discrete, perfect matches could be subject to identification errors during the segmentation step. The discrete map was interpolated using a distance transform function. The epipolar line of each of the guide wire points from the first view is projected on the second view, where the points are evenly distributed along the guide. Then, the distance from each of the epipolar lines and the points in the second view is evaluated and mapped against each other as illustrated in Fig 3. Thereby, it is possible to formulate the correspondence problem as a monotonic search problem in a graph. However, the epipolar lines were calculated using the DICOM meta data of the sequences as an initial estimation of the calibration parameters. Therefore, any error in the calibration necessarily introduces an error in the distance map. The marked beads identified during the segmentation phase are used as anchor points to correct the distance map. For a point $\mathbf{x}_{j}^{\prime}$ and the two nearest knows correspondences at the Euclidean distance $d_{1}$ and $d_{2}$ and where their respective shift with their epipolar line is $\Delta_{1}$ and $\Delta_{2}, \mathbf{x}_{j}^{\prime}$ is translated by:

$$
\Delta_{\mathbf{x}_{j}^{\prime}}=-\left(\Delta_{1}\left(1-\frac{d_{1}}{d_{1}+d_{2}}\right)+\Delta_{2}\left(1-\frac{d_{2}}{d_{1}+d_{2}}\right)\right)
$$

Hence, the closer a point $\mathbf{x}_{j}^{\prime}$ is to one of the anchor points, the more the weight of this matching is important in the calculation of the point shift. Once the shift is calculated for the all the points, a new position for each point is obtained:

$$
\hat{\mathbf{x}}_{j}^{\prime}=\mathbf{x}_{j}^{\prime}+\Delta_{\mathbf{x}_{j}^{\prime}} .
$$

A new map is calculated using those corrected positions and the optimal matching was obtained by finding the shortest path through the graph using Dijkstra shortest path algorithm. 


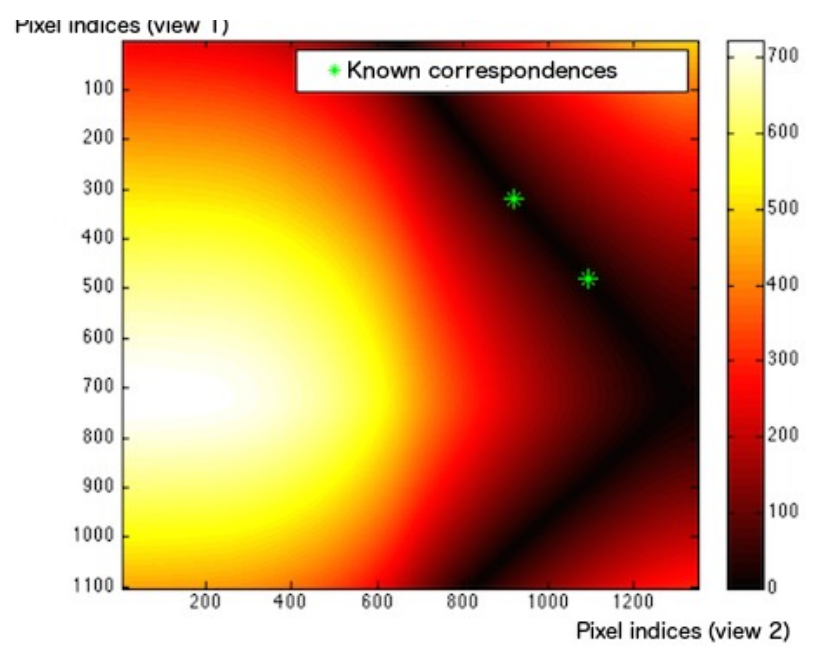

Figure 3: Establishment of the stereo correspondence using a distance map. The darker area represents matching pixels while the green landmarks are manually annotated correspondence.

\subsection{Iterative optimization of the C-arm parameters}

Intrinsic and extrinsic parameters of the C-arm were initialized using the DICOM metadata. From this initial solution, an iterative optimization scheme was introduced to find the optimal parameters. The objective function was the mean 2D error between the segmented guide wire or catheter and the projection of the 3D reconstruction of the elongated structure of the catheter and guide wire and is formulated as follows:

$$
\min \boldsymbol{F}\left(R, \vec{t}, S I D_{j}, c_{j, x}, c_{j, y}\right)=\sum_{i=1}^{n} \sum_{j=1}^{2}\left\|\mathbf{x}_{j, i}-P_{j} \widetilde{\mathbf{X}}_{i}\right\|^{2}+\varpi \sum_{i=1}^{n-1} \sum_{j=1}^{2}\left\|\frac{\mathbf{x}_{j, i+1}-\mathbf{x}_{j, i}}{\left\|\mathbf{x}_{j, i+1}-\mathbf{x}_{j, i}\right\|}-\frac{P_{j} \widetilde{\mathbf{X}}_{i+1}-P_{j} \widetilde{\mathbf{X}}_{i}}{\left\|P_{j} \widetilde{\mathbf{X}}_{i+1}-P_{j} \widetilde{\mathbf{X}}_{i}\right\|}\right\|^{2},
$$

with $R, \vec{t}, S I D_{j}, c_{j, x}, c_{j, y}, \varpi$, being a 3D rotation matrix, a translation vector, the Source Image Distance, the center of projection in $x$ and $y$ and a weighing parameter used to control the influence of the second factor. This function was optimized using the Levenberg-Marquardt algorithm and the parameters was bound to the vicinity of their original value. After each round of optimization, the correspondences are recalculated and the overall optimization process is executed until the convergence of the established correspondences and of the optimized parameters.

\section{RESULTS AND DISCUSSION}

The proposed method was validated using simulated $3 \mathrm{D}$ and $2 \mathrm{D}$ guide wire data, with known projective information and known 3D coordinates. The simulations were conducted under different levels of Gaussian distributed noise (up to 10 degrees or $10 \mathrm{~mm}$ ) applied to the parameters. They have shown a reduction of the mean 2D RMS retroprojection error $(0.061 \mathrm{~mm})$ and a distance to the epipolar line under machine precision.

Evaluation of the method was also performed using retrospective fluoroscopic sequence from pediatric cardiac patients treated for pulmonary stenosis. Overall mean RMS retro projection error on identified points after optimization is in average $0.38 \mathrm{~mm}$ and the mean the distance to the epipolar line is below machine precision. The uncalibrated and calibrated results for each patients are shown in Tab. 1 for the quantitative results and the qualitative results are shown in Fig. 4. After calibration, the matching is significantly improved between the landmarks and the epipolar lines. In addition of the marked catheter, which might offer only a few visible landmarks, the tip of the guide wire was used. For patient 2 and 4 (Fig. $4 \mathrm{~b}$ and d), the tip of the guide wire is successfully realigned with the epipolar line. For instance, this alignment would be highly interesting for locating and reconstructing sequentially the tip of the guide wire in 3D for navigation. 

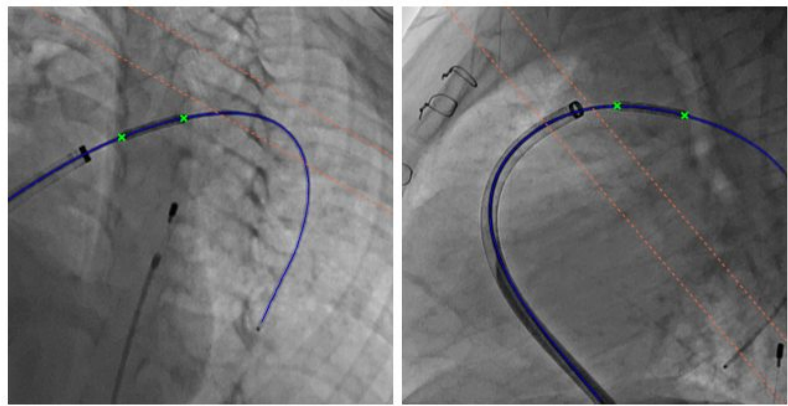

(a)
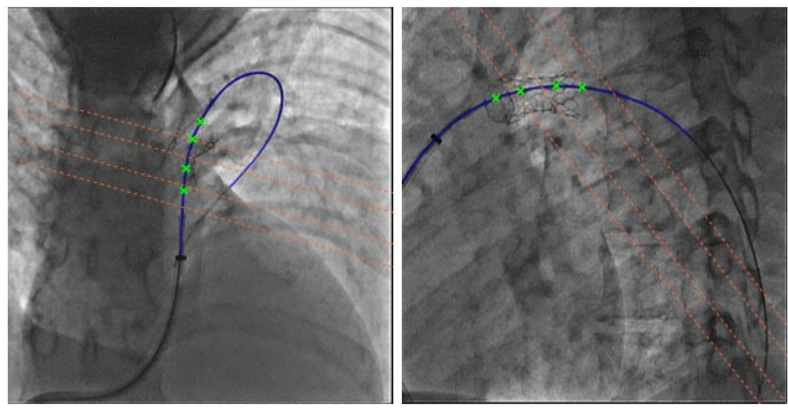

(c)
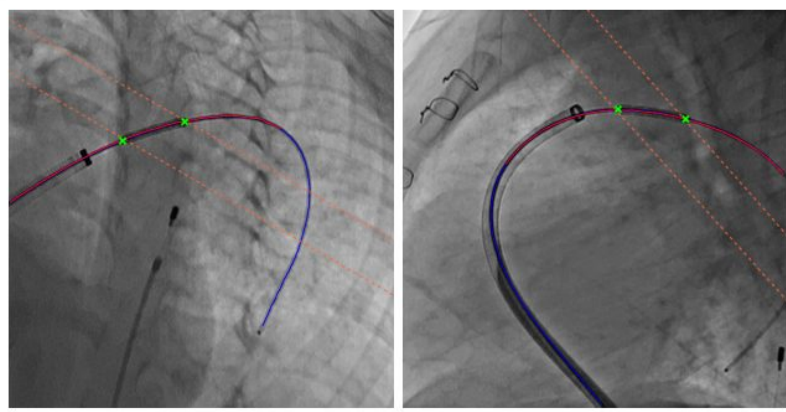

(b)
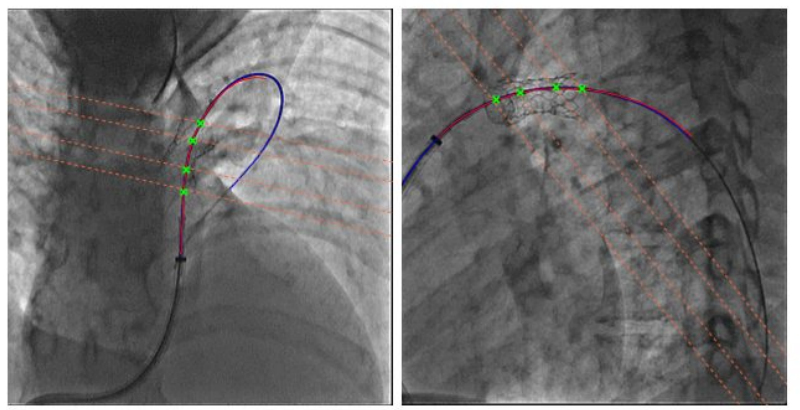

(d)

Figure 4: Pair of fluoroscopic images before (a-c) and after (b-d) the calibration of 2 different patients. The green landmarks illustrate known correspondences and the matching epipolar line is depicted in orange.

Table 1: Quantitative evaluation of the calibration errors using patient datasets

\begin{tabular}{|c|c|c|}
\hline Patients & Before Calibration (2D RMS mm) & After Calibration (2D RMS mm) \\
\hline Patient 1 & 0.90 & 0.17 \\
\hline Patient 2 & 1.27 & 0.26 \\
\hline Patient 3 & 3.37 & 0.50 \\
\hline Patient 4 & 2.25 & 0.60 \\
\hline
\end{tabular}

\section{CONCLUSION}

This study presented a novel framework for online correction of calibration provided by DICOM parameters using the elongated shape of instrumentation such as guide wire and catheter inserted in the patient during treatment of pulmonary stenosis. This is appealing since the presence of a object, with known dimensions within the targeted lesion should contribute to verify the accuracy and robustness of the calibration. Calibration using the entire sequence promises to improve the overall robustness of measurements, to identify drift in the calibration given by the C-arm manufacturer in the DICOM headers and finally can be used for projective geometry applications, such as augmented reality as discussed in Jain et al. ${ }^{11}$ Online calibration of C-arm system could help determine when the system requires a full calibration procedure and contribute to improved 3D reconstruction of vascular structures without adding additional steps to the clinical workflow. Future works would involve automating the catheter and guide wire identification and investigating whether the sequential calibration of the whole fluoroscopic sequence might be relevant to improve robustness of C-arm calibration.

\section{REFERENCES}

[1] Moer, R., van Weert, A. W., Myreng, Y., and Mølstad, P., "Variability of quantitative coronary angiography: an evaluation of on-site versus core laboratory analysis," The international journal of cardiovascular 
imaging 19(6), 457-464 (2003).

[2] Cheriet, F. and Meunier, J., "Self-calibration of a biplane x-ray imaging system for an optimal three dimensional reconstruction," Computerized medical imaging and graphics 23(3), 133-141 (1999).

[3] Yang, J., Wang, Y., Liu, Y., Tang, S., and Chen, W., "Novel approach for 3-d reconstruction of coronary arteries from two uncalibrated angiographic images," IEEE Transactions on Image Processing 18(7), 1563$1572(2009)$.

[4] Liao, R., Duong, L., Sun, Y., and Kirchberg, K., "3-d reconstruction of the coronary artery tree from multiple views of a rotational x-ray angiography," International journal of cardiovascular imaging 26(7), 733-749 (2010).

[5] Rougee, A., Picard, C. L., Trousset, Y. L., and Ponchut, C., "Geometrical calibration for 3d x-ray imaging," in [Medical Imaging 1993], 161-169, International Society for Optics and Photonics (1993).

[6] Kerrien, E., Vaillant, R., Launay, L., Berger, M.-O., Maurincomme, E., and Picard, L., "Machine precision assessment in 3d/2d digital subtracted angiography image registration," in [Medical Imaging'98], 39-49, International Society for Optics and Photonics (1998).

[7] Hartley, R. and Zisserman, A., [Multiple view geometry in computer vision], Cambridge university press (2003).

[8] Weickert, J., "Coherence-enhancing diffusion filtering," International Journal of Computer Vision 31(2-3), 111-127 (1999).

[9] Frangi, A. F., Niessen, W. J., Vincken, K. L., and Viergever, M. A., "Multiscale vessel enhancement filtering," in [International Conference on Medical Image Computing and Computer-Assisted Intervention], 130-137, Springer (1998).

[10] Siddiqi, K., Bouix, S., Tannenbaum, A., and Zucker, S. W., "Hamilton-jacobi skeletons," International Journal of Computer Vision 48(3), 215-231 (2002).

[11] Jain, A. K., An, M., Chitphakdithai, N., Chintalapani, G., and Fichtinger, G., "C-arm calibration: is it really necessary?," in [Proc. SPIE], 6509, 65092U-65092U-16 (2007). 